\title{
ANTONIO BANFI E LE FILOSOFIE DELLA GERMANIA DEL NOVECENTO
}

\author{
Stefano Poggi ${ }^{1}$
}

\begin{abstract}
RIASSUNTO: Tra le figure più importanti del dibattito filosofico italiano del Novecento, Antonio Banfi ha svolto nell'Italia del secondo dopoguerra anche un ruolo politico di rilievo come senatore del PCI. La sua interpretazione del marxismo ha presentato una forte accentuazione umanistica. Tra i suoi scolari filosofi e storici della filosofia come Giulio Preti, Enzo Paci, Remo Cantoni, Paolo Rossi. Il saggio prende in esame la prima fase della riflessione filosofica di Banfi, nella quale ha una importanza decisiva la conoscenza diretta del dibattito tedesco tra le due guerre mondiali, in primo luogo della fenomenologia di Husserl e della ontologia di N. Hartmann. I Principi di una teoria della ragione libro apparso alla fine degli anni '20 - e poi una serie di incisivi saggi degli anni '30 documentano una conoscenza approfondita e critica di un dibattito di cui mostrerà di nutrirsi in misura decisiva l'interpretazione di Banfi non solo di Hegel, ma anche di Marx.
\end{abstract}

PAROLE CHIAVE: Filosofia e politica in Italia nel secondo dopoguerra. Fenomenologia di Hegel e fenomenologia di Husserl. Ontologia e critica dell'esperienza. Marxismo.

1. In tutto il suo svolgimento, il pensiero di Antonio Banfi è segnato da un continuo confronto con il dibattito filosofico della Germania del Novecento. Prima attraverso l'insegnamento di Piero Martinetti, poi - e ben presto - in virtù d'un contatto diretto avviatosi nella Berlino degli anni di poco precedenti il primo conflitto mondiale e proseguito poi ininterrottamente fino alla fine degli anni Trenta la Germania del Novecento con le sue filosofie viene a costituirsi non solo in punto di riferimento, ma in vero e proprio nucleo propulsivo del pensiero di Banfi. E anche se gli anni della guerra e del

\footnotetext{
${ }^{1}$ Nato a Firenze (1947). Professore Ordinario di Storia della Filosofia, Università di Roma e poi Firenze, dal 1981. Pubblicazioni: I sistemi dell'esperienza. Psicologia, logica e teoria della scienza da Kant a Wundt. Bologna: Il Mulino, 1977; Introduzione al positivismo. Roma-Bari: Laterza, 1987; Gli istanti del ricordo. Memoria e afasia in Proust e Bergson. Bologna: Il Mulino, 1991; Il genio e l'unità della natura. La scienza della Germania romantica 1790-1830. Bologna: Il Mulino, 2000; La logica, la mistica, il nulla. Una interpretazione del giovane Heidegger. Pisa: Le edizioni della Normale 2006; La vera storia della Regina di Biancaneve. Dalla Selva Turingia a Hollywood. Milano: Cortina, 2007; L'io dei filosofi e l'io dei narratori. Da Goethe a Proust. Milano: Cortina, 2011; L'anima e il cristallo. Alle radici dell'arte astratta. Bologna: Il Mulino, 2014.
} 
dopoguerra vedranno restringersi gli spazi riservati alla riflessione filosofica, nondimeno sarà sempre il pensiero tedesco a rappresentare per Banfi un termine di confronto essenziale.

L'ampiezza dei documenti a disposizione rende tutt'altro che difficile ricostruire e analizzare il lungo rapporto di Banfi con un dibattito filosofico che è tanto ricco quanto complesso. Vi è anzi la possibilità di farlo sin nei più minuti dettagli, in ciò aiutati dall'ampiezza e dalla puntualità dei riferimenti alla produzione filosofica della Germania tra la fine del secolo XIX e i primi tre decenni del secolo XX presenti negli scritti di Banfi. A onor del vero talvolta i riferimenti sono impliciti, ma in ogni caso l'impresa è sotto ogni profilo del tutto fattibile. La sua realizzazione richiede solo del tempo, pur se con il rischio concreto di disperdersi in un'analisi delle "fonti".

Le obiezioni di chi sostenesse l'inutilità di principio di questa indagine potrebbero forse apparire troppo forti, inutilmente radicali. In ogni caso, ad esse è possibile controbattere con buoni motivi: l'utilizzazione serrata e razionale dei risultati di una indagine che a taluni può apparire puramente e semplicemente antiquaria aiuta a cogliere alcuni dei nodi più interessanti e peculiari della riflessione di Banfi e soprattutto dà modo di afferrarne e apprezzarne la densità, la ricchezza e la stessa attualità.

2. Gli interessi del giovane Banfi nella direzione del dibattito filosofico tedesco trovano più d'uno stimolo nell'insegnamento di Piero Martinetti. Ciò vale innanzitutto per la dichiarata necessità d'una vera e propria teoria metafisica del conoscere che, incentrata sulla ricognizione del nesso tra mondo e coscienza, ha nella filosofia kantiana il suo nucleo propulsivo e da questa è guidata a dare rilievo centrale alla riflessione sul carattere della libertà umana. Le indicazioni che in proposito possiamo ricavare dalle pagine dedicate a Martinetti in quella che rimane l'opera di Banfi più densa e massiccia - e cioè i Principi d'una teoria della ragione del $1926^{2}$ - non lasciano spazio ad equivoci, pur se Banfi non pare comunque condividere quella sorta di monadologia che ispira in molti casi la riflessione di Martinetti e ne mette in evidenza i legami solo apparentemente singolari con le visioni del tardo positivismo.

È però l'impatto diretto con il mondo filosofico tedesco a rivelarsi determinante - e non c'è da meravigliarsene, visto che tale impatto ha luogo

${ }^{2}$ BANFI, A. Principi di una teoria della ragione [1926]. Roma: Editori Riuniti, 1967. In seguito BANFI, [1926] 1967. 
nella Berlino del 1910 - per la maturazione degli orientamenti filosofici di Banfi, così come, trascorsi gli anni della guerra, prendono a delinearsi negli scritti degli anni Venti: l'introduzione alla traduzione degli Hauptprobleme der Philosophie di Simmel, il ciclo di conferenze intitolato La filosofia e la vita spirituale e infine i già ricordati Principi. ${ }^{3}$

Si potrebbe essere tentati di accostare quest'ultimo testo al notissimo libro di Georges Gurvitch del 1929 dedicato alle tendenze dominanti nella filosofia tedesca del tempo. ${ }^{4}$ L'analogia - fatto salvo il diritto di precedenza di Banfi, che pubblica il suo lavoro nel 1926 - può reggere. Ambedue i testi documentano la centrale importanza del dibattito tedesco per la filosofia europea dei primi decenni del Novecento e l'interesse con cui a tale dibattito si guarda da parte di culture e di tradizioni diverse. Ma è anche vero che laddove il lavoro di Gurvitch si contraddistingue per una impostazione fondamentalmente espositiva, esegetica, quello di Banfi presenta invece un carattere molto più problematico. Merita seguire Banfi in alcuni dei punti più rilevanti di tale discussione - in primo luogo, ma non solo nei Principi.

3. Le posizioni di Martinetti costituiscono in ogni caso termine di riferimento fondamentale per il giovane Banfi. In esse Banfi ravvisa la formulazione più "organica" e "trasparente" della esigenza di una teoria del conoscere incentrata sulla "trascendentalità della sintesi conoscitiva e del principio della ragione". ${ }^{5}$ Per una teoria del conoscere di questo tipo è essenziale muovere dalla constatazione non solo che il "fatto della conoscenza" è costituito dall' "accedere di un fenomeno all'aggregato di stati coscienti che è l'Io", ma anche che "tale fenomeno" è uno stato cosciente, che partecipa "della stessa natura della coscienza in cui rientra".

Anche se in termini diffidenti nei confronti della propensione di Martinetti per alcune idee facilmente riconducibili al Mikrokosmos di Lotze, è nondimeno l'analisi della dimensione coscienziale a impegnare il giovane

\footnotetext{
${ }^{3}$ L'introduzione (con il titolo: Il relativismo critico e l'intuizione filosofica della vita) alla traduzione di Simmel e il ciclo di conferenze La filosofia e la vita spirituale sono rispettivamente del 1922 e del 1921. I testi ora (rispettivamente alle p. 275-300 e 2-160) in BANFI, A. Opere, I: La filosofia e la vita spirituale e altri scritti di filosofia e religione (1919-1929), a cura di Luciano Eletti con la collaborazione di Livio Sichirollo, Istituto Antonio Banfi-Regione Emilia Romagna, s.i.l., 1986. In seguito Banfi 1986.

${ }^{4}$ GURVITCH, G. Les tendances actuelles de la philosophie allemande. Paris: Vrin, 1929.

${ }^{5}$ BANFI, [1926] 1967, p. 302.
} 
Banfi, preoccupato di assicurare la fondazione e lo svolgimento del processo conoscitivo in costante interazione con la medesima. È questa la prospettiva di un razionalismo trascendentale $e^{6}$ fondato sul primato del problema conoscitivo e della sua soluzione in forza della fondamentale autonomia dello "spirito" come unità di vita ed esperienza non cristallizzata su di un piano metafisico. ${ }^{7}$ Si tratta di un convincimento inequivocabilmente ispirato da alcuni motivi conduttori della discussione tedesca a Banfi contemporanea, segnata nel profondo da una esigenza anti-riduzionistica, ma convinta che la salvaguardia di tale esigenza non debba comportare restaurazioni metafisico-spiritualistiche.

Non è difficile rendersi conto di ciò dinanzi all'affermazione dell'importanza delle "analisi gnoseologiche ed epistemologiche di senso unitario". La distinzione tra gli aspetti più propriamente fenomenologici del conoscere e la legge trascendentale che, "espressione della pura esigenza teoretica", si vorrebbe che presieda ai medesimi attraverso la "sintesi correlativa di soggetto e oggetto" è in effetti una distinzione tipica di quanto maturato in ambito neokantiano e fenomenologico, e in termini più o meno convinti del ruolo più o meno paradigmatico delle scienze. Banfi mostra di essere decisamente convinto delle ragioni di quello che indica come il "trascendentalismo tedesco" (dizione sotto cui colloca il neokantismo di Marburg, la variegata teorizzazione - a dominante componente neokantiana - intorno ai valori e quindi la stessa fenomenologia husserliana). Per Banfi - vicino al dibattito della filosofia tedesca anche per quanto riguardo l'interpretazione della filosofia antica (Natorp, Stenzel, Prantl, Gomperz) - la prospettiva di un razionalismo trascendentale appare in grado di contemperare - con ben altra energia della debole speculazione positivista - le esigenze di una fondazione della conoscenza guidata dai metodi e dai dati delle scienze con quelle della individualità conoscitiva e pratica dell'uomo. ${ }^{8}$

4. Il privilegio accordato da Banfi alla filosofia tedesca si conferma anche negli anni successivi alla pubblicazione dei Principi d'una teoria della ragione, allorché - rimanendo fermo l'interesse per la fenomenologia di Husserl Banfi accentua il proprio interesse per i problemi legati all'estetica filosofica,

${ }^{6}$ BANFI, [1926] 1967, p. 232-235.

7 PAPI, F. Il pensiero di Antonio Banfi. Firenze: Parenti, 1961 (in seguito PAPI, 1961), p. 132-133.

${ }^{8}$ BANFI, [1926] 1967, p. 235, 396ss., 309ss., 369ss. 
con un occhio di riguardo per il confronto non poco innovativo con i risultati della ricerca psicologica tipico del dibattito tedesco dell'epoca. ${ }^{9}$

Motivo conduttore della riflessione di Banfi rimane comunque l'adesione ad un razionalismo critico e trascendentale a un tempo che assegna una specifica rilevanza alla dimensione coscienziale nella misura in cui non pare ammettere la riduzione di quest'ultima al piano degli eventi cosiddetti fisici. Nello stesso tempo, è il nodo del rapporto tra religione e metafisica a presentarsi come d'importanza particolare. In Banfi - che tra l'altro è curatore negli stessi anni di una raccolta di scritti di Böhme - emerge una attenzione per il fenomeno religioso nelle sue valenze non confessionali che è tipica della cultura del tempo: si pensi all'interesse per l'intricato complesso della mistica e delle varie forme della esperienza religiosa legato alla fortuna dell'omonimo libro di W. James. ${ }^{10}$ Su questa linea, i riferimenti di Banfi al dibattito della filosofia tedesca del tempo toccano sì questioni relative agli studi religiosi e alla loro sistematica, ma soprattutto riguardano un problema dalle innegabili implicazioni filosofiche come quello della immanenza e della trascendenza. ${ }^{11}$

È d'altronde anche in questo caso la dimensione della coscienza a presentarsi a Banfi come questione di rilievo centrale: lo conferma l'attenzione delle conferenze del 1921 intorno alla vita spirituale e poi degli scritti che seguono fino agli anni '30 per l'intreccio psicologia-empiriocriticismo. Banfi è dell'avviso che il fenomenismo "alla Mach" assegni un rilievo non giustificabile alla sensazione. Facendo per un verso proprio l'antipsicologismo dello Husserl delle Logische Untersuchungen e per un altro condividendo quella che appare una correzione delle posizioni di Mach avanzata da Ziehen, Banfi contrappone al critero machiano della economia del pensiero la fondamentale sistematicità di quest'ultimo e afferma che la sensazione, "[...] presentata come soluzione immediata della problematicità del conoscere, non può porsi come la sfera

\footnotetext{
${ }^{9}$ Si vedano le recensioni raccolte in BANFI, 1986, p. 359-378. Si veda inoltre il testo Sui principi di una filosofia della morale [1934] in BANFI, A. Opere, X, La ricerca della realtà [1959], a cura di Guido Davide Neri e Gabriele Scaramuzza con la collaborazione di Barbara Cavalieri, 2 tomi, Istituto Banfi-Società Editrice il Mulino 1996, s.i.l. (in seguito: BANFI, 1996), p. 493 ss. Cf. anche BANFI, A. I problemi di un'estetica filosofica [1932-1933] e L'esperienza estetica e la vita dell'arte [1940] rispettivamente alle p. 5ss e 76ss di: BANFI, A. Opere, V: Vita dell'arte. Scritti di estetica e filosofia dell'arte, a cura di E. Mattioli e G. Scaramazza con la collaborazione di L. Anceschi e D. Formaggio, Istituto Banfi-Regione Emilia Romagna (in seguito BANFI, [1959] 1988.

${ }^{10}$ BANFI, A. Per il congresso del libero cristianesimo [1910], in Banfi 1986, p. 165-179.

${ }^{11}$ BANFI, A. Immanenza e trascendenza come antinomia filosofica [1924], ora in BANFI, 1986, p. 180 192. Cf. anche BANFI, [1959] 1996, II, p. 649-675, 650-651, 661.
} 
immediata dell'essere". La posizione di Banfi è chiara, nel suo far proprie alcune specifiche tesi maturate in seno al dibattito intorno ai vari programmi di scientificizzazione della psicologia. ${ }^{12}$ I caratteri della sfera psicologica non possono essere quelli della "[...] mera, astratta soggettività" ${ }^{13}$ È però anche vero che il carattere costitutivamente dialettico della "speculazione psicologica" impone di prendere atto della debolezza di ogni punto di vista psicologicoassociazionistico, inerme dinanzi ad una dinamica - quella soggetto-oggetto ovvero dell'io e del non-io - che è invece costitutiva della vita psichica. Ciò non significa però il passaggio allo spiritualismo: la critica filosofica da esercitare nei confronti della psicologia - Banfi è vicino a molte posizioni neokantiane ${ }^{14}$ - impone di prendere atto che la coscienza, connotando "l'essere psicologico come tale", corrisponde sì "ad una posizione ideale dello spirito", ma non può imporre - come "[...] forma universale dell'esperienza"15 - di concepire il mondo "sotto l'aspetto della coscienza". Se i dati della analisi psicologica hanno un loro peso, ciò non mette in discussione che "[...] il pensiero filosofico è il superamento della posizione soggettiva della coscienza conoscitiva, è l'idealità universalmente posta”. ${ }^{16}$

5. La convinzione del carattere dialettico della dinamica coscienziale si rafforza con l'incontro di Banfi con la psicologia fenomenologica: Scheler, Binswanger, già prima Dilthey. ${ }^{17}$ Banfi manifesta un vivo interesse al riguardo, rivelatore di una impostazione di riflessione filosofica assai mobile, attenta alla costante interazione tra strutture della coscienza e strutture d'una razionalità dagli evidenti caratteri polimorfi.

Sotto questo angolo visuale, le ragioni della vicinanza di Banfi a Simmel sono evidenti, anche se sono necessarie alcune importanti puntualizzazioni, come ancor più nel caso del rapporto di Banfi con la fenomenologia husserliana. Come si è rilevato da più parti - ma tra i primi ad affermarlo con chiarezza era stato Fulvio Papi ${ }^{18}$ - lo Husserl di Banfi è e rimane fondamentalmente quello

\footnotetext{
${ }^{12}$ BANFI, [1959] 1996 II, p. 533-534.

${ }^{13}$ BANFI, [1959] 1996, II, p. 535ss.

${ }^{14}$ BANFI, [1926] 1967, p. 251 sgg. Ma cf. anche p. 237.

${ }^{15}$ BANFI, [1926] 1967, p. 252-253.

${ }^{16}$ BANFI, [1926] 1967, p. 120, p. 138.

${ }^{17}$ BANFI, [1959] 1996, p. 526-527.

${ }^{18}$ PAPI, 1961, p. 98ss.
} 
delle Logische Untersuchungen, della Philosophie alsstrenge Wissenschaft e di Ideen $I$ e, per quanto Banfi possa seguire anche la pubblicazione dello "Jahrbuch für Phänomenologie und phänomenologische Forschung" fino al $1928^{19}$, di fatto il rapporto con la fenomenologia husserliana rimane cristallizzato nei termini in cui esso si configura nei Principi del 1926, dove ad emergere come punti fondamentali di riferimento sono da un lato l'indipendenza della sfera logica, dall'altro la purezza della coscienza. La tesi della importanza centrale per Husserl di una "sistematicità teoretica generale" finisce poi per offuscare il carattere dinamico della struttura intenzionale della coscienza a favore della intuizione. E tutto questo - è forse superfluo aggiungerlo - si rivela non particolarmente funzionale allo sviluppo di quella linea di riflessione che, pronta ad accogliere suggestioni di varia provenienza, ha in Simmel il suo vero e proprio catalizzatore.

Sulla funzione catalizzatrice delle diverse linee di riflessione presenti sulla scena tedesca svolta da Simmel sarebbe quanto mai opportuno soffermarsi. In tale prospettiva, è da tenere presente innanzitutto la già ricordata introduzione di Banfi alla traduzione degli Hauptprobleme der Philosophie del 1922. Banfi - che accanto agli Hauptprobleme mostra di avere presente la Einleitung in die Moralwissenschaft e poi anche la Philosophie des Geldes - vede nel relativismo di Simmel una concezione fondamentalmente relazionale del pensiero. Pur senza rendere esplicito (e forse senza neanche cogliere) il retroterra herbartiano di Simmel, Banfi ravvisa in quest'ultimo temi a lui di fatto già noti per il tramite di Martinetti: il relativismo è lo "sforzo estremo di dare un significato alle parzialità e alle opposizioni dei sistemi metafisici". È vero che l' "infinita progressività dell'esigenza teoretica" fa sì che il "processo soggettivo" finisca col cedere alle ragioni di "una oggettività il cui sistema formale rende possibile l'introduzione dei contenuti più differenti", ma è vero anche nello stesso tempo che permane il dato di una "vita spirituale" come tensione interiore contraddistinta da una costituzionale polarità imperniata sull'individuo come centro di "tutti i processi spirituali". È la nozione stessa di individuo a chiedere di essere precisata e approfondita, pur se è incolmabile la distanza tra gli effettivi contenuti del nostro conoscere (e della stessa nostra coscienza) ed il significato ideale che i medesimi devono essere in grado di ricevere per potere essere oggetto del conoscere e del pensare. Tale distanza è conclamata nel caso dell'azione morale, il cui "fatto ultimo" è in ogni modo impensabile "come dato" e il cui valore presenta una idealità assoluta che è l'autentica

${ }^{19}$ PAPI, 1961, p. 99. 
"categoria metafisica della individualità", individualità nella quale si realizza il massimo di universalità. Posto dinanzi "alle parzialità e alle opposizioni dei sistemi metafisici", l'individuo è sì obbligato alla scelta del relativismo per dare un significato a tali "parzialità" e a tali "opposizioni", ma trova anche nella possibilità di trascendere la vita - il "fenomeno originario della vita" consiste infatti nel potere realizzare il "superamento di sé stessa" - la soluzione della contrapposizione tra assoluto e relativo. ${ }^{20}$

6. Non c'è bisogno di aggiungere molto altro. Il retroterra - per un verso kantiano per un altro tardo-positivistico (se così va intesa la vicinanza ad alcuni motivi della visione di Lotze) - da cui muove Banfi allievo di Martinetti mostra di essere potentemente fecondato dal contatto con una prospettiva filosofica - appunto quella di Simmel - in cui rilievo primario è assunto dalle modalità di costituzione e di espressione dell'individuo sia per quanto riguarda la dinamica conoscitiva sia per quanto invece tocca - nel nodo della coscienzialità in cui tutto si intreccia - la sfera dell'agire. Da una parte dunque la costituzionale autotrascendenza della vita che assicura l'individuo di quella che potremmo dire la sua sopravvivenza ideale, dall'altra la forza di quella dialettica che rompe le contrapposizioni polari cui si trova esposto l'individuo e fornisce così una sorta di visione panoramica di un processo altrimenti spezzettato e desultorio. Quel che così si era delineato in Simmel era il passaggio alla prospettiva hegeliana, con una significativa attenzione per il concreto, per una individualità nella quale ha sede e motivo propulsore a un tempo quella operazione protesa al superamento della contrapposizione tra assoluto e relativo che è l'agire, l'agire morale come operazione che ha il suo punto di leva nel principio dell' autocoscienza.

Le tracce di tutto questo nelle posizioni di Banfi sono nitidissime. L'attenzione per il nucleo costituito dall'individuo autocosciente centro di una potenzialità dialettica è il segno, in Banfi, di un interesse sempre più spiccato per la visione hegeliana, interesse che è indizio chiarissimo della persistente reattività - grazie non solo alla mediazione di Simmel - di Banfi alle linee di tendenza del dibattito della Germania del tempo. Sempre in sintonia con il "basso continuo" dell'impegno sul piano del non-riduzionismo dello "spirito", Banfi assegna un rilievo decisivo alla dimensione non solo del conoscere, ma

${ }^{20}$ BANFI, 1986, p. 275-300. 
anche a quella del valutare e dell'agire, e in termini che sottintendono un confronto con Kant e il neokantismo.

7. Le posizioni di Banfi quali si delineano ad alcuni anni di distanza dai Principi nello scritto del 1934 intitolato Principi di una filosofia della morale sono il segno di una più specifica attenzione per quella che possiamo indicare come la problematica del valore. Tali segni potevano essere in effetti ravvisati già nelle conferenze del 1921 dedicate al rapporto tra filosofia e "vita spirituale" e nell'interesse per le tesi della Philosophie des Geldes di Simmel, attraverso cui Banfi entra in contatto con un intreccio problematico essenziale nella vicenda filosofica tedesca dei primi decenni del secolo XX. È tutta la questione del valore ad essere d'importanza essenziale per la filosofia tedesca 1900-1930 (si pensi, tra l'altro, alla centralità che essa assume nella riflessione di Lukács, e non solo del giovane Lukács). La questione investe in modo diretto il rapporto tra il soggetto (individuo) e l'azione, e nello stesso tempo viene posta in termini che intendono operare costruttivamente nella direzione di una demetafisicizzazione dei fondamenti e dei criteri dell'intera sfera valutativa, là dove si formulano giudizi etici come anche giudizi estetici ed economici. La questione - come si vede innanzitutto nei caratteri assunti dal rinnovato interesse per le questioni fondazionali dell'estetica - emerge e si mette a fuoco d'altronde in un contesto intriso anche degli interrogativi posti dal contrasto logica-psicologia.

La ripresa del trascendentalismo kantiano era apparsa per molti aspetti come la soluzione delle aporie al centro della gigantomachia psicologistica, come per certi aspetti si verifica - per menzionare il caso più noto - proprio con l'opzione in senso trascendentale della fenomenologia husserliana. Ma oltre che a quest'ultima, Banfi guarda anche in altre direzioni, come è il caso - ciò accade nei Principi del 1926 - delle tesi avanzate da O.Külpe contro i pericoli di un panlogismo tendenzialmente esposto all'astrattismo dogmatico dell'idealismo. Tale attenzione è rivelatrice di un interesse centrale per il processo del conoscere e dell'agire come processo cui presiedono criteri di valutazione dei quali è l'individuo e solo questi a portare la responsabilità. Se dunque - nello scritto del 1934 sui Principi di una filosofia della morale, che, detto per inciso, è dedicato "a Edmund Husserl per il suo $75^{\circ}$ compleanno" - Banfi può dichiararsi per un’indagine di filosofia della morale "[...] il cui indirizzo sia puramente teoretico" e muovere nella direzione di una "sorta di idea trascendentale di moralità" con la quale riuscire a "indicare il problema 
che sottostà a tutta la vita morale" 21 , nello stesso tempo emerge anche la fondamentale importanza di un approccio dialettico alla morale, con la rinuncia ad ogni "astratto ideale pragmatico" e la conseguente "[...] scoperta e formulazione dell'idea, come legge della problematica della moralità" ${ }^{22}$

8. L'attenzione che Banfi - nel medesimo scritto del 1934 - dedica alla critica di Scheler al formalismo etico e, con questa, quella alla teoria materiale dei valori non è dunque di circostanza. ${ }^{23}$ La critica di Scheler appare per molti aspetti essere lo sviluppo e la sistematizzazione delle vedute di Simmel circa i fondamenti e le prospettive dell'azione morale come azione di un individuo. ${ }^{24}$ Nondimeno, la conoscenza da vicino delle tesi di Scheler di cui Banfi dà prova non è in alcun modo il segno di una piatta convergenza con le medesime. È d'altronde questo il tratto tipico dell'atteggiamento di Banfi dinanzi al dibattito filosofico della Germania contemporanea, dibattito che è occasione e stimolo di prima importanza, mai però repertorio di soluzioni preconfezionate. Un caso per certi aspetti emblematico è quello già evocato di O. Külpe e della teoria da questi avanzata d'una Realisierung; accanto a questo si potrebbero menzionare anche le tesi di Nicolai Hartmann, da Banfi accolte come tesi con cui guardare, senza cedimenti a dogmatismi metafisici o empirici, alle categorie dell'essere come "[...] espressione dell'immediatezza formale della ragione". ${ }^{25}$

Tutto questo è vero. Ma, da un punto di vista generale, è vero anche che il confronto di Banfi con il dibattito filosofico della Germania a lui contemporanea può apparire - in forza di una sorta di imprinting ricevuto con l'insegnamento di Piero Martinetti - sostanzialmente come una rimodulazione delle originarie formulazioni kantiane, sulla linea di una tesi interpretativa tipicamente e decisamente neokantiana (i nomi di Riehl e di Cassirer non sono taciuti da Banfi! ${ }^{26}$ ) come quella per cui - così le pagine iniziali de La filosofia e la vita spirituale ${ }^{27}$ - la "grande scoperta kantiana" consiste nell' "aver riconosciuto

${ }^{21}$ BANFI, [1959] 1996, II, p. 498ss, 501.

22 BANFI, [1959] 1996, II, p. 504-505.

23 BANFI, 1986, p. 396-405 e poi BANFI, [1959] 1996, II, p. 518-520, 523-526, 528, 530, 532, 553, 555. Cf. PAPI, 1961, p. 99, 205, 252, 335.

${ }^{24}$ BANFI, [1959] 1996, p. 518-520.

${ }^{25}$ BANFI, [1926] 1967, p. 291-293, 303, 301.

${ }^{26}$ BANFI, [1926] 1967, p. 304ss.

${ }^{27}$ BANFI, 1986, p. 13-14. 
nel carattere formale e indipendente dalla particolarità del proprio contenuto" che connota le "forme d'unità dei corpi o dei fenomeni naturali" il vero e proprio "principio d'organicità e di verità della scienza". Sarebbe poi possibile con altrettanti buoni motivi ravvisare nell'insistenza con cui Banfi - ciò vale d'altronde soprattutto per i Principi del 1926 - sottolinea l'esigenza del punto di vista d'una razionalità trascendentale la conferma di una più che sostanziale fedeltà al dettato kantiano anche là dove tale punto di vista pare potere essere consolidato dalla fenomenologia di Husserl. Ciò avviene d'altronde in linea con quanto matura presso molti esponenti neokantiani, nel quadro di una convergenza di fondo nell'indagine circa i modi in cui il soggetto conoscente è in possesso di forme, di strutture a priori costitutive di fatto di quella che viene indicata come realtà.

9. Anche il crescente interesse di Banfi per l'agire morale parrebbe doversi collocare su questa linea. Tuttavia, pur sottolineando che l'esperienza morale kantiana è come un' "esperienza trasfigurata" in cui si dà un vero e proprio "albeggiare di spiritualità" ${ }^{28}$, Banfi si trova nondimeno condotto a riconoscere una certa qual rigidità del formalismo etico kantiano. A ciò si aggiunge anche la presa d'atto di un altro aspetto problematico della filosofia kantiana: quello per cui - tiene a sottolineare Banfi in termini che lo collocano in una posizione oggettivamente distante da non piccola parte dell'esegesi neokantiana - il rischio di un "annullamento della varietà infinita della esperienza” pare essere implicito nell'atteggiamento di Kant per cui "[...] l'esperienza è fissata in forma univoca, dogmatica, secondo un sistema staticamente concluso di forme categoriali-teoretiche". ${ }^{29}$

Le ragioni per cui - come d'altronde già accennato - in Banfi emerge più di un segno di attenzione per l'altra fonte fondamentale del dibattito della Germania a lui contemporanea e cioè per la filosofia di Hegel sono a questo punto non solo evidenti, ma anche dense di implicazioni. ${ }^{30}$ Banfi - in ciò va vista un'ulteriore conferma del distacco da quanto in Martinetti permane della visione di Lotze e della stessa tradizione herbartiana, peraltro spesso contrapposta a quella hegeliana - tende anzi ravvisare proprio in quella che è a suo avviso la tendenziale rigidità con cui Kant prospetta il nesso conoscere-

\footnotetext{
${ }^{28}$ BANFI, [1926] 1967, p. 356-357.

${ }^{29}$ BANFI, [1926] 1967, p. 356

${ }^{30}$ PAPI, 1961, p. 188ss, 193, 201.
} 
essere una delle radici stesse del panlogismo ${ }^{31}$ anche hegeliano. Banfi non esita ad affermare che la logica hegeliana ricade nell' "errore kantiano" quando assume come costitutive dell'esperienza le categorie scientifiche ricavate "[...] da una determinata situazione dogmatico-logica". ${ }^{32}$

La già evocata presa di distanza dal formalismo etico kantiano e quindi il rilievo da dare alla dimensione della coscienza con quel che in essa è tanto implicito quanto potentemente iscritto di dinamicità storica sono dunque i due motivi propulsivi di fondo che non possono non spingere Banfi a guardare con una crescente attenzione a Hegel, ma a guardarlo - si è visto con una esplicita avversione nei confronti del panlogismo e del sistema. Non è però puramente e semplicemente l'appello alla dinamicità del processo vitale intrecciata con quella della storia in una intrinseca dinamica teleologica che in Banfi si presenta come un motivo guida di un confronto innovatore con la filosofia hegeliana restituita alle sue radici autentiche dalla nascente HegelPhilologie. La rilevanza primaria del problema della coscienza e dell'individuo significa per Banfi anche volgere lo sguardo là dove la concezione hegeliana opera proprio per sottolineare, pur salvaguardandone la piena concretezza, in qual misura la coscienza dell'individuo sia la sede di un contrasto tra la moralità della persona e l'ordine etico della società, viva insomma in sé medesima di una vita che è fatta di scissioni e di contrasti. ${ }^{33}$

10. Il richiamo alla Phänomenologie des Geistes che emerge nelle pagine dei Principi del 1926 non deve allora sorprendere. Non sono moltissime, invero, quelle pagine e non sempre il riferimento al capolavoro hegeliano è diretto, esplicito. Ma quel che in esse colpisce è come Banfi - che alla Phänomenologie si accosta muovendo dal suo solidissimo background tedesco e prima della comparsa di quei lavori interpretativi che di quell'opera avrebbero fatta e al tempo stesso condizionata la fortuna per molti decenni del Novecento - sia netto e preciso nella sua caratterizzazione del compito che Hegel aveva assegnato al testo del 1807: assicurare la certezza del conoscere e, con essa,

\footnotetext{
${ }^{31}$ BANFI, [1926] 1967, p. 359

${ }^{32}$ BANFI, [1926] 1967,p. 363-364, 367.

${ }^{33}$ BANFI, [1959] 1996, II, p. 506 (è il saggio Sui principi di una filosofia della morale del 1934): la sintesi morale come sintesi ideale di una dualità antinomica. Nel medesimo saggio, su Hegel le p. 510-512 e 514-515, dove viene trattato anche del contrasto tra moralità della persona e ordine etico della società: Rousseau e l'idealismo di Fichte e di Hegel. E ancora p. 553 nota sul rapporto naturamoralità in Hegel.
} 
quella del soggetto autocoscienze che opera a tutti i livelli di una realtà causa e nello stesso momento effetto del suo agire e del suo pensare. Non è dunque solo la lettura di Hegel - con il privilegio accordato al primo Hegel - proposta da Dilthey e poi da Nohl ad essere ben presente a Banfi, che già negli anni di Berlino aveva potuto conoscere il celebre saggio di Windelband Die Erneuerung des Hegelianismus del 1910 e, ovviamente, ha ben presente la ricostruzione di Kroner, Von Kant bis Hegel apparsa all'inizio degli anni Venti. ${ }^{34}$ Da queste letture, dal suo contatto diretto con il milieu filosofico tedesco in cui sono in pieno sviluppo il "rinnovamento" ovvero la "rinascita" della filosofia hegeliana Banfi è sollecitato a guardare alla filosofia hegeliana non solo come al nucleo propulsore di quella "filosofia della vita" di cui avverte la potente suggestione, ma anche come concezione complessiva della realtà che ne coglie l'autentica dinamica propulsiva, e la ravvisa nella ricognizione del processo nel corso del quale si manifesta, sin dal livello della certezza che può essere assicurata dai sensi, l'intrinseca razionalità dialettica del pensiero. ${ }^{35}$ È uno Hegel che per molti aspetti non appare particolarmente vicino alle immagini più o meno forzate o comunque parziali che verranno ad accreditarsi nel corso del secolo XX e in special modo in anni molto difficili per l'Europa; è uno Hegel che se si volesse cedere al criterio dei "precorrimenti" potrebbe addirittura apparire assai vicino all'immagine che oggi ne vige nella filosofia del mondo anglosassone e, di riflesso, in quella dell'intero mondo occidentale; è - in una parola - uno Hegel che è una delle figure decisive del pensiero moderno, e che innanzitutto come tale appare a Banfi, che non esitava a indicarlo come il primo dei suoi grandi maestri, ${ }^{36}$ quello da cui aveva potuto attingere insegnamenti decisivi per mettere a punto il proprio senso di distanza dalle grossolanità dello scientismo positivista, senza per questo cedere alla simmetrica arroganza dell'idealismo nazionale e quindi alla sommaria liquidazione di ogni proficuo rapporto tra la riflessione filosofica e la conoscenza scientifica.

\footnotetext{
${ }^{34}$ WINDELBAND, W. Die Erneuerung des Hegelianismus. Heidelberg: Winter, 1910; KRONER, R. Von Kant bis Hegel. Tübingen: Mohr, 1921-1924, 2v.; Cf. BANFI, [1926] 1967, p. 172.

${ }^{35}$ Cf. BANFI, [1926] 1967, p. 90.

${ }^{36}$ BANFI, [1959] 1996, p. 700.
} 
11. È in una pagina molto nitida dei Principi di una teoria della ragione che questa consapevolezza di Banfi si manifesta senza margini di equivoco, nella sottolineatura della distanza di Hegel dalla "posizione misticodogmatica” di Schelling. Banfi teneva a sottolineare la consapevolezza con cui lo Hegel della Phänomenologie des Geistes aveva affermato che la filosofia, per "[...] avere carattere di scienza e definire l'ordine della realtà", deve "[...] disporsi nell'organicità di un sistema". Questo sistema è il "sistema universale della ragione, in cui la coincidenza del pensiero e dell'essere si sviluppa in una complessa organicità di posizione”. Esso è un sistema “[...] che ha il suo fondamento non in uno di tali momenti, ma nella legge unitaria di tutti i momenti stessi" così come si dispiega sin a partire dal livello elementare del "[...] dualismo proprio della coscienza determinata", coscienza che - scriveva Banfi - è “[...] immediato essere in sé dell'universalità teoretica” ${ }^{37}$ quale si dà sul piano della naturale immediatezza d'una riflessione psicologica la cui certezza in realtà "implica ed esprime, in funzione della sua particolare posizione, l'universalità autonoma della ragione". "Universalità autonoma della ragione" che "[...] giunge a dispiegarsi libera" in quel "sistema della scienza" la cui essenza costitutiva è di essere "[...] legge di correlatività e di movimento". ${ }^{38}$ Banfi non aveva esitazione a ricordare e a condividere, citando letteralmente il testo della Phänomenologie des Geistes, la tesi hegeliana per cui la "[...] vera forma, in cui la verità esiste, può essere solo il suo sistema scientifico" e poi la ribadiva, sottolineandone l'esplicita ispirazione anti-fichtiana, con l'affermazione che "[...] il sapere è reale e può essere espresso solo come scienza, o come sistema”, dato che comunque il sapere - e quello filosofico in particolare - è tale solo in virtù della universalità, idealità e quindi oggettività della "[...] reciproca corrispondenza dei suoi elementi" ${ }^{39}$ E dunque solo la "struttura razionale della realtà" ${ }^{\prime 0}$ che così viene a delinearsi ad assicurare l'effettiva, reale oggettività della conoscenza della medesima. Non solo più che a Dilthey e a Nohl, ma anche più che a Windelband e a Kroner, le pagine dei Principi di una teoria della ragione dedicate a Hegel mostravano di ispirarsi a quanto era stato affermato già nel 1906 da un grande storico della filosofia come Ernst Cassirer, che più di tanti hegeliani studiosi di Hegel era stato in grado di cogliere e mettere in luce la coerenza con cui Hegel, nella Phänomenologie des

\footnotetext{
${ }^{37}$ BANFI, [1926]1967, p. 25, n.1.

${ }^{38}$ BANFI, [1926]1967, p. 360-362.

${ }^{39}$ BANFI, [1926]1967, p. 177 e poi 180.

${ }^{40}$ BANFI, [1926]1967, p. 361.
} 


\title{
Geistes, aveva inteso realizzare "[...] lo sviluppo e l'esposizione completa del problema dell'oggettività." ${ }^{41}$
}

POGGI, Stefano. Antonio Banfi and 19th century German philosophy. Trans/Form/Ação, Marília, v. 37, n. 3, p. 201-216, Set./Dez., 2014.

\begin{abstract}
Among the leading philosophers in Italy during the 20th century, Antonio Banfi played an important role not only in the cultural debate during the 1930s and the 1940s, but also in the political scene of post-war Italy, advocating - as a representative of the Italian Communist Party in Italian Senate - a liberal, humanist view of Marxism. Among his students were influential philosophers and historians of philosophy such as Giulio Preti, Enzo Paci, Remo Cantoni and Paolo Rossi. This paper investigates the first phase of Banfi's philosophical development, an analysis of which must include a scrutiny of Banfi's deep indebtedness to German debate between the two World Wars, primarily Husserl's phenomenology and Hartmann's critical ontology. Banfi's phenomenological apprenticeship at the school of Husserl, and the sensitive and critical attitude of his presentation of the German debate in his Principi di una teoria della ragione, are of substantial importance in nourishing his later interpretation of Hegel and Marx.
\end{abstract}

KEYWORDS: Italian philosophy and politics. Husserl's and Hegel's phenomenology. Ontology and critique of experience. Marxism.

\section{BibLIOGRAFIA}

BANFI, A. Principi di una teoria della ragione [1926]. Roma: Editori Riuniti, 1967.

BANFI, A. La filosofia e la vita spirituale e altri scritti di filosofia e religione (1919-1929). In:__ Opere. A cura di Luciano Eletti con la collaborazione di Livio Sichirollo. Reggio nell'Emilia: Istituto Antonio Banfi; [Bologna]: Regione Emilia Romagna, 1986. v.1.

La ricerca della realtà [1959]. In: Opere. A cura di Guido Davide Neri e Gabriele Scaramuzza con la collaborazione di Barbara Cavalieri. Reggio nell'Emilia: Istituto Antonio Banfi; Bologna: Il Mulino, 1996. v. 10, 2 tomos.

- Vita dell'arte. Scritti di estetica e filosofia dell'arte. In: Opere. A cura di E. Mattioli e G. Scaramazza con la collaborazione di L. Anceschi e D. Formaggio. Reggio nell'Emilia: Istituto Antonio Banfi; [Bologna]: Regione Emilia Romagna, 1988. v. 5.

CASSIRER, E. Storia della filosofia moderna. Trad. di E. Arnaud. Torino: Einaudi, 1955.

${ }^{41}$ CASSIRER, E. Storia della filosofia moderna. Trad. di E. Arnaud. Torino: Einaudi, 1955 sgg. (Das Erkenntisproblem in der Philosophie und Wissenschaft der neueren Zeit. Berlin: Bruno Cassirer 1906ss. 3v.), v. 3: Il problema della conoscenza nei sistemi postkantiani. Torino: Einaudi, 196133, p. 413. 
POGGI, S.

GURVITCH, G. Les tendances actuelles de la philosophie allemande. Paris: Vrin, 1929. KRONER, R. Von Kant bis Hegel. Tübingen: Mohr, 1921-1924. 2v.

PAPI, F. Il pensiero di Antonio Banfi. Firenze: Parenti, 1961.

WINDELBAND, W. Die Erneuerung des Hegelianismus. Heidelberg: Winter, 1910.

Recebido em: 14/09/2014

Aceito em: 29/09/2014 